Pour ce qui est des autres élements, voici les re- $\mid$ zone de $5 \mathrm{~m}$ d' AR. et, de 15' de largeur, je n'ai rensiltats moyens. contré que la h. 1802 , et une autre étoile jaune $=\mathbf{7 . 0}$ qui passe environ 3 in 3 après (ce qui correspondrait en gros avec l' AR. donnée par les M. M. pour la 2942) et que je suppose être l'étoile solitaire que j'ai observé en 1863 et 1864 .

Ensuite j'ai examiné expres le ciel, mais dans nne

Le trois positions dans l'espace données par $\mathrm{h}, \boldsymbol{\Sigma}$ et $\mathrm{O} \Sigma$, reduites à 1873 donneraient:

\begin{tabular}{|c|c|c|c|}
\hline $\mathrm{h}=22^{\mathrm{h}} 38^{\mathrm{m}} 19^{\mathrm{s}}$ & D. P. $=51^{\prime \prime} 11^{\prime} .5$ & N. 1802 & IV. Cat. \\
\hline$\Sigma=$ & 5111.3 & 2942 & Hon. Frid. 18. M. N \\
\hline$\Sigma=2238 \quad 17$ & 5111.8 & 478 & Cat. Rev. de 1850. \\
\hline
\end{tabular}

La coincidence des trois distances Polaires est vraiment frappante. - Je crois qu'en admettant une erreur de $+2 \mathrm{~m}$ dans l'AR. de $\Sigma$, l'identité de la double 2942 est plus que prouvée.

Pour les mesures anterieures, voir les Astr. Nachr. Vol. 62, 66, 73, 75, 79.

Gallarate, le 2. Janvirir 1874.

\title{
Beobachtungen der Flora (8) auf der Moskauer Universitäts-Sternwarte.
}

Die Messungen wurden mit erleuchteten Fäden und bei einer Vergrüsserung von 300 ausgefihrt. Die Anzahl der Beobachtungen ist sehr gering wegen der schlechten Witterung. - Da die Vergleichsterne 9.5 Grösse in unserem Refructor sehr schwach e schienen, so war die Pointirung ziemlich schwierig, was auch in den Grössen der wahrscheinlichen Fehler sich zeigt.

Die vorliegenden Declinationsdifferenzen sind schon vou dem Einfluss der Refraction befreit.

\begin{tabular}{|c|c|c|c|c|c|c|c|}
\hline \multirow[t]{5}{*}{1873} & October & 26 & $\begin{array}{c}\text { Mosk. mittl. Zt. } \\
\begin{array}{ll}13^{\text {h }} 17 \mathrm{~m} & 0 \text { s. } 2\end{array}\end{array}$ & $\begin{array}{l}\text { (8) - * } \\
+2^{\prime} 11^{\prime \prime} .375\end{array}$ & $\begin{array}{l}\text { Wahrsch. } \\
\text { Fehler. } \\
+0^{\prime \prime} .143\end{array}$ & $\begin{array}{c}\text { Anzahl } \\
\text { d. Vergl. } \\
12\end{array}$ & $\begin{array}{l}\text { * Nummer } \\
\text { d. Bonn. Verz. } \\
+2^{0}(410)\end{array}$ \\
\hline & 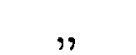 & 27 & 1115 & +017.757 & \pm 0.084 & 10 & $f-2(412)$ \\
\hline & , & 27 & $11 \cdot 37$ & -15.715 & \pm 0.195 & 6 & $(410)$ \\
\hline & 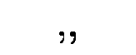 & 31 & 1356 & +42.334 & \pm 0.102 & 12 & $(402)$ \\
\hline & $n$ & 31 & $13 \quad 55 \quad 28.1$ & -534.666 & \pm 0.089 & 12 & $+2(408)$ \\
\hline
\end{tabular}

Moskau, den 20. Februar 1874.

Prof. Dr. Th. Brediılin.

\section{Schreiben des Herrn Prof. Wats on an den Herausgeber.}

I have discovered an error in the computation of the ephemeris of Helena (101) given by me in the Astronomische Nachrichten No. 1968, and I subjoin a corrected ephemeris computed by means of the elements there published.
The ephemeris includes the perturbations by $\mathrm{J}_{11}$ piter and Saturn, and since it agrees quite well with the observed places at the present opposition it appears that my elements are pretty nearly correct. 